\title{
Exploration of dynamical regimes of irradiated small protonated water clusters
}

\author{
Uguette Flore Ndongmouo Taffoti ${ }^{1}$, Phuong Mai Dinh ${ }^{2}$, Paul-Gerhard Reinhard ${ }^{2,3}$, Eric Suraud ${ }^{2}$, and Zhi Ping \\ Wang $^{4}$ \\ 1 CEA Saclay, DEN/SRMP, F-91191 Gif/Yvette Cedex \\ 2 Laboratoire de Physique Théorique, IRSAMC, UPS and CNRS, Université de Toulouse, 118 Rte de Narbonne, F-31062 \\ Toulouse cedex, France \\ ${ }^{3}$ Institut für Theoretische Physik, Universität Erlangen, Staudtstrasse 7, D-91058 Erlangen, Germany \\ 4 School of Science, JiangNan University, Wuxi 214122, China
}

First draft: oct 2009

\begin{abstract}
We explore from a theoretical perspective the dynamical response of small water clusters, $\left(\mathrm{H}_{2} \mathrm{O}\right)_{n} \mathrm{H}_{3} \mathrm{O}^{+}$with $n=1,2,3$, to a short laser pulse for various frequencies, from infrared (IR) to ultraviolet (UV) and intensities (from $6 \times 10^{13} \mathrm{~W} / \mathrm{cm}^{2}$ to $5 \times 10^{14} \mathrm{~W} / \mathrm{cm}^{2}$ ). To that end, we use time-dependent local-density approximation for the electrons, coupled to molecular dynamics for the atomic cores (TDLDA$\mathrm{MD}$ ). The local-density approximation is augmented by a self-interaction correction (SIC) to allow for a correct description of electron emission. For IR frequencies, we see a direct coupling of the laser field to the very light $\mathrm{H}^{+}$ions in the clusters. Resonant coupling (in the UV) and/or higher intensities lead to fast ionization with subsequent Coulomb explosion. The stability against Coulomb pressure increases with system size. Excitation to lower ionization stages induced strong ionic vibrations. These maintain rather harmonic pattern in spite of the sizeable amplitudes (often $10 \%$ of the bond length).
\end{abstract}

PACS. 3 6.40.Mr -3 6.40.Vz -3 1.15.ee $-3 \quad 3.60 .+\mathrm{q}$

\section{Introduction}

The structure of water is generally considered to be rather ordered due to hydrogen bonds and with certain structures prevailing. There is, in turn, a general agreement among models about the existence of small and mediumsized clusters of water molecules [1. Furthermore, experimental evidence point to the fact that under appropriate conditions, water clusters may ionize. There thus exists, since rather long, a large body of investigations on charged water clusters, either anions [2/3/4, cations [6] or protonated clusters 788 .

The dynamics of water clusters appears as a key issue in various scenarios, e.g., for the chemistry of formation of droplets and clouds in atmosphere 9. Water clusters are also possible candidates as transient intermediate stages in liquid water [10. They might thus play a key role in many chemical and physical processes 11/12/3 14/15. The case of charged clusters, and especially ionized clusters, is particularly relevant for studies on radiation damage, as water is the natural "environment" of biomolecules. This has triggered recent studies on the influence of a finite number of water molecules coating a biomolecule 16. The direct analysis of the irradiation of protonated water clusters is also a key issue [17.
From the theoretical point of view, water clusters have been considered since long at a structural level [2|4|5, for a recent summary see 18. In recent years, studies of irradiation of biomolecules, possibly in a water environment have also been started [1920, but usually without an explicit time-dependent treatment of the ionization process itself. In the present paper, we aim at studying the response of small protonated water clusters to an irradiation by a short laser pulse. The choice of laser irradiation rather than excitation by a charged projectile is to some extent a matter of convenience as it allows for exploratory studies a discrimination and a systematic scan of various dynamical regimes without dealing with complications of scattering geometry. Furthermore, there exists a well documented literature on the laser irradiation of clusters, metallic or rare gas mostly 21/22 in a wide range of excitations, which provides a robust background to study irradiation of other sorts of clusters such as water clusters. The paper is organized as follows. We first briefly recall the basic inputs of the model, in section 2. As a next step we first consider the optical response which is known to provide a key entry point to understand irradiation dynamics (section 3). We then address irradiation by an intense laser filed, exploring in particular the influence of laser frequency and intensity as key characteristics of 
the laser pulse (section 4). We finally draw our conclusions in section 5 .

\section{Model}

In order to perform microscopic simulations of dynamical processes, we employ time-dependent density functional theory (TDDFT) 232425] for the valence electrons (described in terms of single-electron wave functions $\left.\left\{\varphi_{\alpha}(\mathbf{r}), \alpha=1 \ldots N_{\mathrm{el}}\right\}\right)$ combined with classical molecular dynamics (MD) for the ionic cores (described by their classical coordinates $\left.\left\{\mathbf{R}_{I}, I=1 \ldots N_{\text {ion }}\right\}\right)$. The ions are here $\mathrm{H}^{+}$, and $\mathrm{O}^{6+}$. The starting point is an energy which includes the electron kinetic energy, the direct Coulomb energy, the exchange-correlation energy, the coupling energy between electrons and ions (described in terms of non-local pseudo-potentials of the type [26] and tuned to provide a most efficient description on the numerical grid [27]), and the energy coming from the interaction of the system with the external time-dependent laser field coupling to both, electrons and ions. The functional for the electronic exchange-correlation energy is taken at the level of the local-density approximation (LDA) with the actual form from [28. The electronic energy is augmented with an average-density self-interaction correction (ADSIC) 29. The ADSIC is a crucial ingredient to provide correct ionization potentials (IP) which, in turn, is necessary to describe electron emission correctly.

Equations of motion are then derived in a standard manner by variation of the given energy. Variation with respect to $\varphi_{\alpha}$ leads to the (time-dependent) Kohn-Sham equations for the single-particle wave functions. Variation with respect to the ionic coordinates yields Hamiltonian equations of motion for ions, thus treated by classical MD. Electronic and ionic equations of motion are solved simultaneously leading to coupled TDLDA-MD description 3031. The treatment goes thus beyond the adiabatic approximation. It becomes equivalent to the BornOppenheimer (adiabatic) approximation in regimes where the latter is valid.

Time-dependent fields and wave functions are represented on a $3 \mathrm{D}$ coordinate-space grid of dimensions $72 \times$ $72 \times 64$ or $72^{3}$, with a grid spacing of $0.412 a_{0}$. The electronic wave functions are propagated in time by the timesplitting method [32]. The Coulomb problem is solved by a fast Fourier technique 33. The ionic equations of motion are solved using the Verlet algorithm. Absorbing boundary conditions are used to properly remove outgoing electrons 3031. Thus the total number of electrons $N=N(t)$ decreases in time. The number of escaped electrons $N_{\text {esc }}=N(t=0)-N(t)$ is a measure of average ionization.

The electronic ground state wave functions are obtained by damped gradient iteration [34. Ionic ground state configurations are obtained by cooling pseudo-dynamics 35. Results for the structure of a few selected water clusters are shown in figure 1. Gray balls stand for the oxygen atoms and are labelled O1, O2, etc. White ball, labelled $\mathrm{H} 1$, etc., represent the hydrogen atoms. The O-H

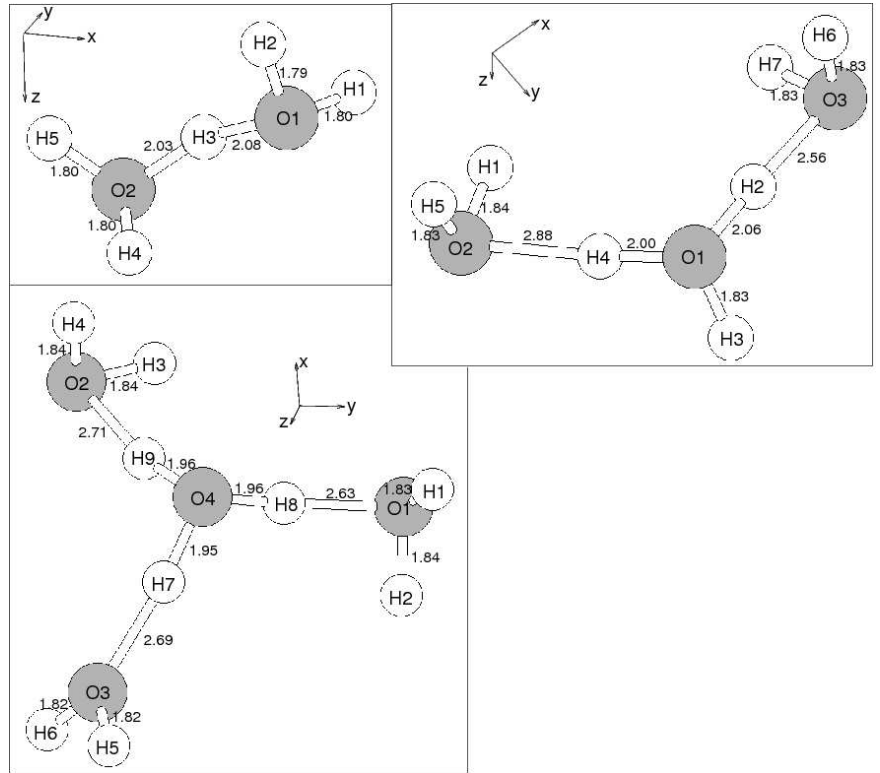

Fig. 1. Schematic view of the initial configuration of the systems $\mathrm{H}_{2} \mathrm{O} \mathrm{H}_{3} \mathrm{O}^{+},\left(\mathrm{H}_{2} \mathrm{O}\right)_{2} \mathrm{H}_{3} \mathrm{O}^{+}$, and $\left(\mathrm{H}_{2} \mathrm{O}\right)_{3} \mathrm{H}_{3} \mathrm{O}^{+}$. Note that all indicated frames are positively oriented.

bond lengths are also indicated and take basically three values, around 1.8, 2.0 and $2.7 a_{0}$.

\section{Optical response}

The spectral distribution of optical absorption strength (optical response) provides key information on the coupling of a system to (laser) light. The initial response is mostly mediated by electrons. The optical response then is related to eigenfrequencies of electronic excitations which exhibit well isolated peaks at frequencies below emission threshold and continuum above. These eigenmodes are important because they represent the doorways for laser excitation. In the case of metal clusters, the major absorption strength lies in the optical domain with a marked collective character. They are known as the Mie plasmon resonances and correspond to a collective oscillation of the electron cloud with respect to the ionic background, with rather well established systematic behaviors with cluster size 3136 . The situation is more involved in organic systems where varying bonding can lead to large differences from one cluster to the next. Furthermore the typical eigenfrequencies lie in the UV part of spectrum rather than in the optical domain and thus require laser pulses with higher frequencies (single photon) or higher intensities (multiphoton processes).

The optical absorption strength can be computed easily within our TDLDA description by the technique of spectral analysis 37/38, for a detailed discussion see [39]. To that end, we apply initially an infinitely short pulse covering all frequencies with equal weight, propagate the system for a sufficiently long time (depending on the desired spectral resolution), record the time-dependent dipole 
momentum $\mathbf{D}(t)$, and finally Fourier transform the signal into the frequency domain. The strength is then obtained as the imaginary part of $\tilde{\mathbf{D}}(\omega)$. Results for opti-

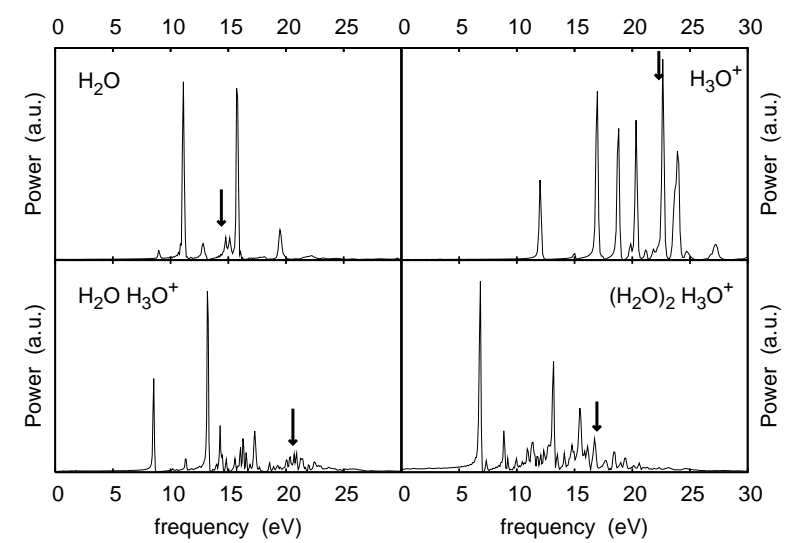

Fig. 2. Spectral distribution of optical absorption strength for the systems $\mathrm{H}_{2} \mathrm{O}, \mathrm{H}_{3} \mathrm{O}^{+}, \mathrm{H}_{2} \mathrm{O} \mathrm{H}_{3} \mathrm{O}^{+}$, and $\left(\mathrm{H}_{2} \mathrm{O}\right)_{2} \mathrm{H}_{3} \mathrm{O}^{+}$. The corresponding IP are indicated by an arrow.

cal absorption spectra of $\mathrm{H}_{2} \mathrm{O}, \mathrm{H}_{3} \mathrm{O}^{+}, \mathrm{H}_{2} \mathrm{O} \mathrm{H}_{3} \mathrm{O}^{+}$, and $\left(\mathrm{H}_{2} \mathrm{O}\right)_{2} \mathrm{H}_{3} \mathrm{O}^{+}$are shown in Figure 2. The spectra exhibit typical structures with well marked and isolated eigenfrequencies at "low" frequencies below the ionization potential (IP) for the given system and a trend to a more fuzzy "continuum" response at higher frequencies above the IP. The figure shows that infrared and visible light is below any significant peak and thus off resonant. Light pulses in the UV range can couple more or less strongly, dependening an the detailed frequency. The figure does also indicate the IP for each system. These are in detail: IP(H2O) $=-14.4 \mathrm{eV}, \operatorname{IP}\left(\mathrm{H}_{3} \mathrm{O}^{+}\right)=-22.3 \mathrm{eV}, \operatorname{IP}\left(\mathrm{H}_{2} \mathrm{O} \mathrm{H}_{3} \mathrm{O}^{+}\right)=$ $-20.6 \mathrm{eV}$, and $\mathrm{IP}\left(\left(\mathrm{H}_{2} \mathrm{O}\right){ }_{2} \mathrm{H}_{3} \mathrm{O}^{+}\right)=-16.9 \mathrm{eV}$.

\section{Irradiation by a strong laser field}

We now turn to direct analysis of the irradiation and response of small protonated water clusters to short and intense laser pulses. Even in the UV regime, the laser wavelength remains much larger than the size of the system under consideration. So the long wavelength limit applies such that the dipole operator describes the spatial part of laser pulse. Thus we model the laser pulse as the time dependent external field

$$
V_{\text {las }}=E_{0} \mathbf{e}_{\text {pol }} \cdot \hat{\mathbf{r}} \cos (\omega t) f(t)
$$

with the amplitude $E_{0}$ being related to the intensity $I$ as $E_{0}\left(\right.$ Ry. $\left.a_{0}^{-1}\right)=1.07 \times 10^{-8} \sqrt{I\left(\mathrm{~W} / \mathrm{cm}^{2}\right)}$ and (linear) polarization $\mathbf{e}_{\text {pol }}$. In all our calculations, we use $\mathbf{e}_{\text {pol }}=\mathbf{e}_{x}$ (see Figure 11). We will see below, in the discussion of figure 4. that the laser polarization has only a limited importance for the basic mechanisms. The laser frequency $\omega$ is carried in the cos term. The pulse profile $f(t)$ is chosen as a $\sin ^{2}$ characterized by its Full Width at Half Maximum (FWHM). In all calculations below we have chosen a FWHM of 20 fs and we have focused on the simple case of $\left(\mathrm{H}_{2} \mathrm{O}\right) \mathrm{H}_{3} \mathrm{O}^{+}$, exploring only at some places larger clusters.

\subsection{Dependence on frequency}

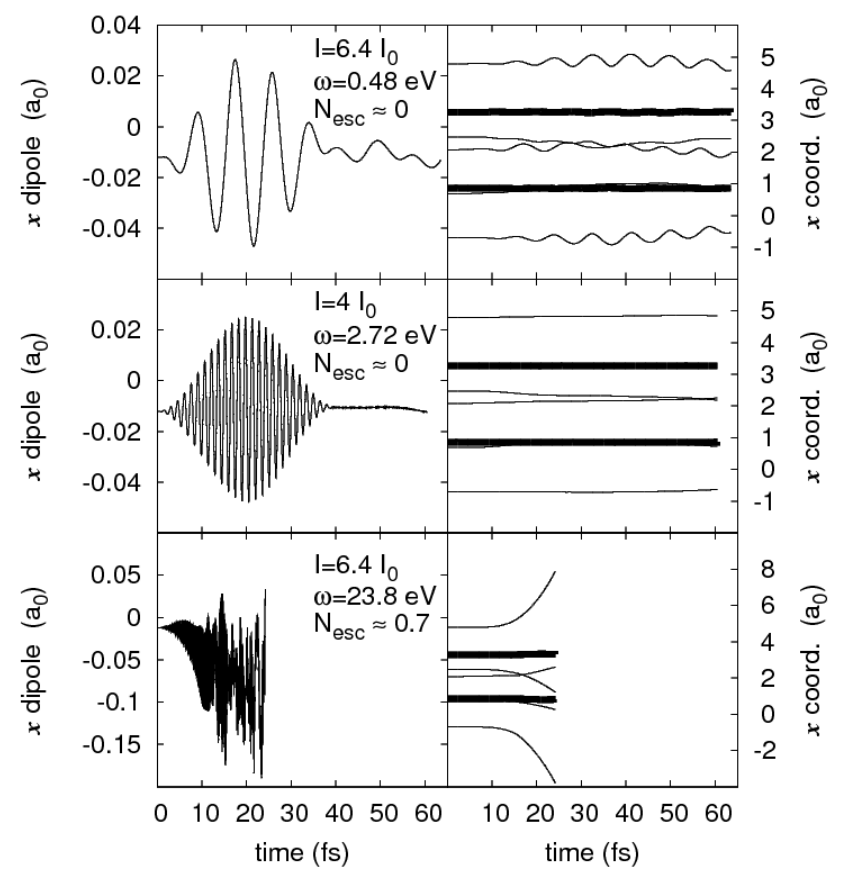

Fig. 3. Irradiation of $\left(\mathrm{H}_{2} \mathrm{O}\right) \mathrm{H}_{3} \mathrm{O}^{+}$by lasers with polarization along $x$ axis, FWHM of $20 \mathrm{fs}$, intensities of about $I_{0}=10^{13}$ $\mathrm{W} / \mathrm{cm}^{2}$, and three frequencies as indicated. The number of escaped electrons $N_{\text {esc }}$ is also reported for each case. As a function of time : Electronic dipole moment in $x$ direction (left column), and ionic $x$ coordinates (right column). The thick curves stand for the position of the oxygen atoms, while the thin lines show the $x$ coordinates of the hygrogen atoms.

We first consider the influence of laser frequency on irradiation dynamics of $\left(\mathrm{H}_{2} \mathrm{O}\right) \mathrm{H}_{3} \mathrm{O}^{+}$, exploring a variation of laser frequency at basically constant laser intensity. Figure 3 shows results for three representative cases: a frequency in the infra-red (IR), another one in the visible range, and an ultra-violet (UV) one. The IR and visible frequencies lie below the range of typical electronic eigenfrequencies in $\left(\mathrm{H}_{2} \mathrm{O}\right) \mathrm{H}_{3} \mathrm{O}^{+}$(see Figure 2), while the UV case lies in the middle of the eigenfrequencies. The overall dynamical behavior is strongly related to the laser frequency. We observe a typical out-of-resonance behavior for IR and visible frequencies where the amplitude of the dipole signal (left column of Figure 3) is proportional to the envelope of the laser field. There is practically no electron emission for the given laser intensity. The UV frequency, being in resonance with some of the systems 
excitation modes, displays a much different behavior: the strong dipole response lasts longer than the laser signal with a sizeable ionization. Correspondingly, the overall behavior of the cluster dynamics behaves quite differently. The right column of Figure 3 displays the time evolution of the ionic positions of constituting ions along the laser polarization axis. While the two off-resonance cases exhibit only low amplitude ionic vibrations, the UV case leads to an almost immediate explosion of the $\left(\mathrm{H}_{2} \mathrm{O}\right) \mathrm{H}_{3} \mathrm{O}^{+}$.

Another interesting aspect concerns the difference between the visible and IR cases. In the frequency in the visible range, one can hardly see any ionic perturbation while the IR irradiation leads to clear ionic vibrations with an small, but not vanishing, amplitude. This difference can be traced back to a direct coupling of the ions (predominantly the hydrogen ions) with the laser itself. This seems a bit surprising in view of the rather small FWHM of the laser pulse (20 fs). But the small hydrogen mass allow such a rapid coupling. This coupling is observed in the IR domain and not for the visible light because typical ionic vibration frequencies in small water clusters precisely lie in the considered IR frequency domain 40. Estimating the $\mathrm{H}$ vibration frequency in this case leads to a value of $\hbar \omega \sim 0.41 \mathrm{eV}$, in fair agreement with previously published results which shows peaks around $0.47 \mathrm{eV}$ [0]. Closer inspection shows that the IR laser pulse simply excites internal vibrations of hydrogen atoms mostly along $\mathrm{OH}$ bonds.

In order to explore this effect in more detail, we now focus on the distances between $\mathrm{O}$ and $\mathrm{H}$ atoms. Indeed, because of their larger masses, $\mathrm{O}$ atoms basically remain fixed during the short time scales explored here and we can thus focus the further analysis on $\mathrm{O}-\mathrm{H}$ bonds only. As can be seen from Figure 1 there are basically two types of $\mathrm{O}-\mathrm{H}$ bonds, the ones between $\mathrm{O}$ and external $\mathrm{H}$ and the internal ones along the sequence $\mathrm{H}-\mathrm{O}-\mathrm{H}$. The associated bond lengths are different and so are the corresponding vibration frequencies. It is thus interesting to consider these two classes of cases separately. The results are plotted in Figure 4. One should first note the remarkable regularity of oscillations, especially for external $\mathrm{H}$ atoms (upper panel), with about all having the same frequency. One also clearly observes oscillations in opposite phases depending on the geometry. This is particularly obvious for the two internal bonds (lower panel of Figure 4). Different heights of amplitudes indicates a different strength of the response which is predominantly a geometry effect due to the orientation of the bond relative to laser polarization, here the $x$ direction. Indeed, the $\mathrm{O}_{1}-\mathrm{H}_{1}$ and $\mathrm{O}_{2}-\mathrm{H}_{5}$ bonds are the external $\mathrm{O}-\mathrm{H}$ bonds which are aligned with the $x$ axis the most (see Figure 1) and which exhibit the largest amplitudes of oscillations. Changing the laser polarization (along the $y$ or the $z$ direction for instance) would produce a largest excitation of other $\mathrm{O}-\mathrm{H}$ bonds but the mechanisms of excitation would remain the same. Finally one should note the amplitude of oscillations which may be rather large, typically $10 \%$ of the actual bond length but without destroying the harmonicity of the oscillations, the latter aspect being especially true for external $\mathrm{H}$ atoms.

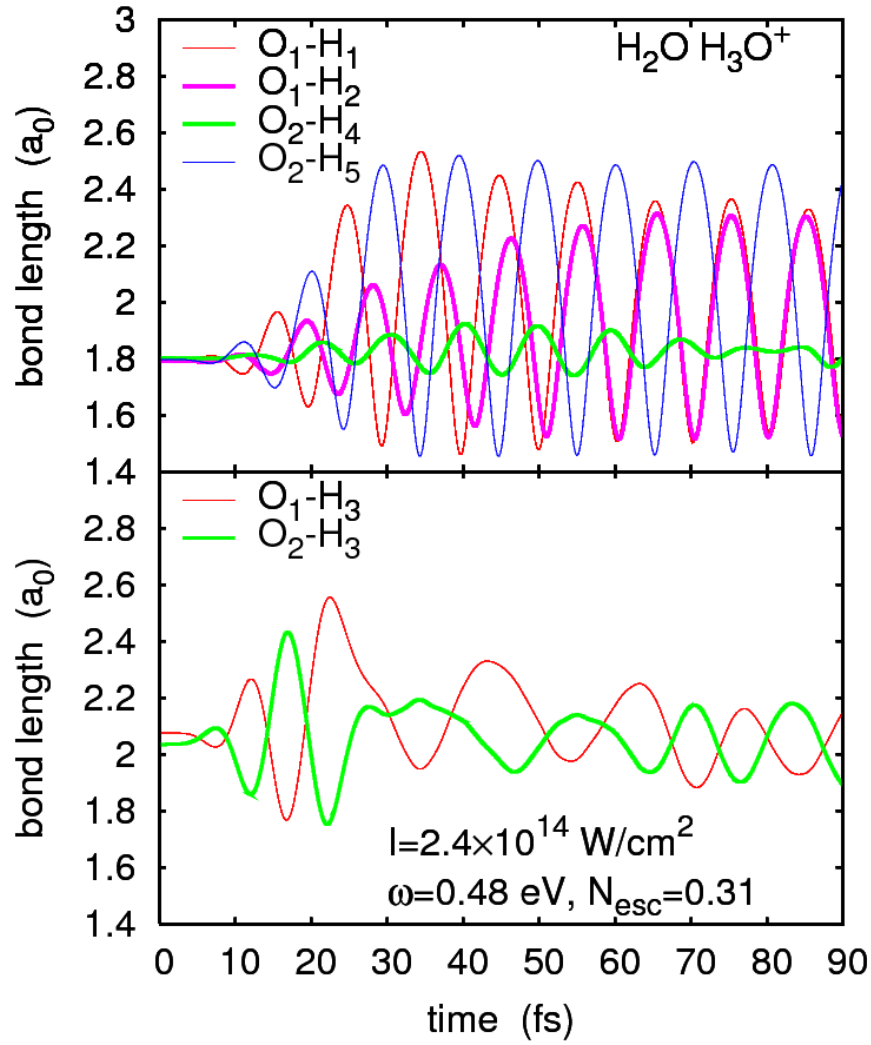

Fig. 4. Irradiation of $\left(\mathrm{H}_{2} \mathrm{O}\right) \mathrm{H}_{3} \mathrm{O}^{+}$by a laser with polarization along $x$ axis, FWHM of $20 \mathrm{fs}$, and intensity and frequency as indicated. The number of escaped electrons is also given. Are plotted as a function of time the $\mathrm{O}-\mathrm{H}$ bond lengths : the shortest ones (top), according to the atom numbering of Figure 1 and the longest ones (bottom).

\subsection{Dependence on intensity}

We have identified in Figure 3 the resonant coupling of the laser pulse with internal IR vibrations along $\mathrm{OH}$ bonds and explored it in more detail in Figure 4. Figure 5 explores how this behavior depends on laser intensity. It shows the time evolution of the distances between the 2 oxygen atoms which lies around $4 a_{0}$ and the typical $\mathrm{OH}$ bonds whose length is about $2 a_{0}$. The influence of laser intensity on the ionic response is obvious. With increasing laser intensity, we observe increasing ionization. Up to about net ionization one, there is little effect on the general vibrational pattern. They gain, of course, amplitude due to the larger field strength. But on the time scales computed here, the systems seems to resist fragmentation and keep its vibrational response. Only for ionization substantially larger than one, the dynamical evolution changes qualitatively to the extent that the now large repulsion leads to an immediate fragmentation of the cluster and, of course, disappearance of the vibrational response. All in all, we thus observe a remarkable robustness of the vibrational response with increasing laser intensity. 


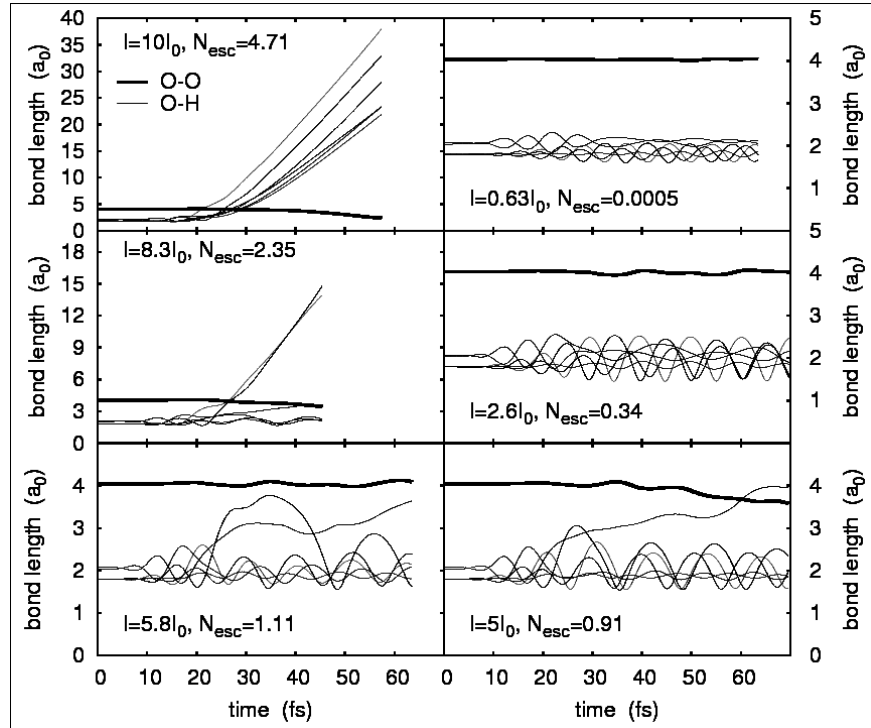

Fig. 5. Irradiation of $\left(\mathrm{H}_{2} \mathrm{O}\right) \mathrm{H}_{3} \mathrm{O}^{+}$by lasers with polarization along $x$ axis, FWHM of $20 \mathrm{fs}$, frequency of $0.48 \mathrm{eV}$, and six different intensities varying about $I_{0}=10^{14} \mathrm{~W} / \mathrm{cm}^{2}$ as indicated. Intensities increase in clockwise order. The average ionization (number of escaped electrons $N_{\text {esc }}$ ) is also indicated for each case. Various bond lengths (O-O and shortest $\mathrm{O}-\mathrm{H})$ are depicted as a function of time.

\subsection{Dependence on size}

It is finally interesting to explore how dynamical evolution depends on cluster size. We consider here the case of $\left(\mathrm{H}_{2} \mathrm{O}\right)_{n} \mathrm{H}_{3} \mathrm{O}^{+}$clusters for $n=1,2,3$. We again take the same IR frequency at $0.48 \mathrm{eV}$ and a moderate laser intensity to remain in the vibrational regime in $\left(\mathrm{H}_{2} \mathrm{O}\right) \mathrm{H}_{3} \mathrm{O}^{+}$. Various $\mathrm{OH}$ bond lengths are plotted as a function of time in Figure 6. Because of the quickly increasing number of $\mathrm{OH}$ bonds with increasing size, which makes a figure including all of them unreadable, we have selected a few representative cases, following the labelling given in Figure1 Note that, although we use the same laser in all cases, the net ionization differs from one case to the next, due to different ionization potentials: $-20.6 \mathrm{eV},-16.9 \mathrm{eV}$, and $-15.7 \mathrm{eV}$ for $n=1,2$, and 3 respectively; associated net ionizations are $0.34,1.44,2.00$ respectively for $n=1,2,3$. This should not be a problem as we have seen that the effects are to a large extent robust with net ionization. Indeed, at least on the short time scale we have considered here, we observe in all cases comparable vibrational response, in spite of the large ionization in the largest cluster. Note, however, that the larger ionization appears in the larger system which has naturally a higher capability to cope with a high charge state. Such an effect has been found also for the Coulomb fragmentation of metal clusters 41 .

Figure [6] exhibits interesting features in detail. The upper panel compares typical external $\mathrm{O}-\mathrm{H}$ vibrations in the $n=2$ and $n=3$. In both clusters, the corresponding bonds have very similar lengths and frequencies, as is clear

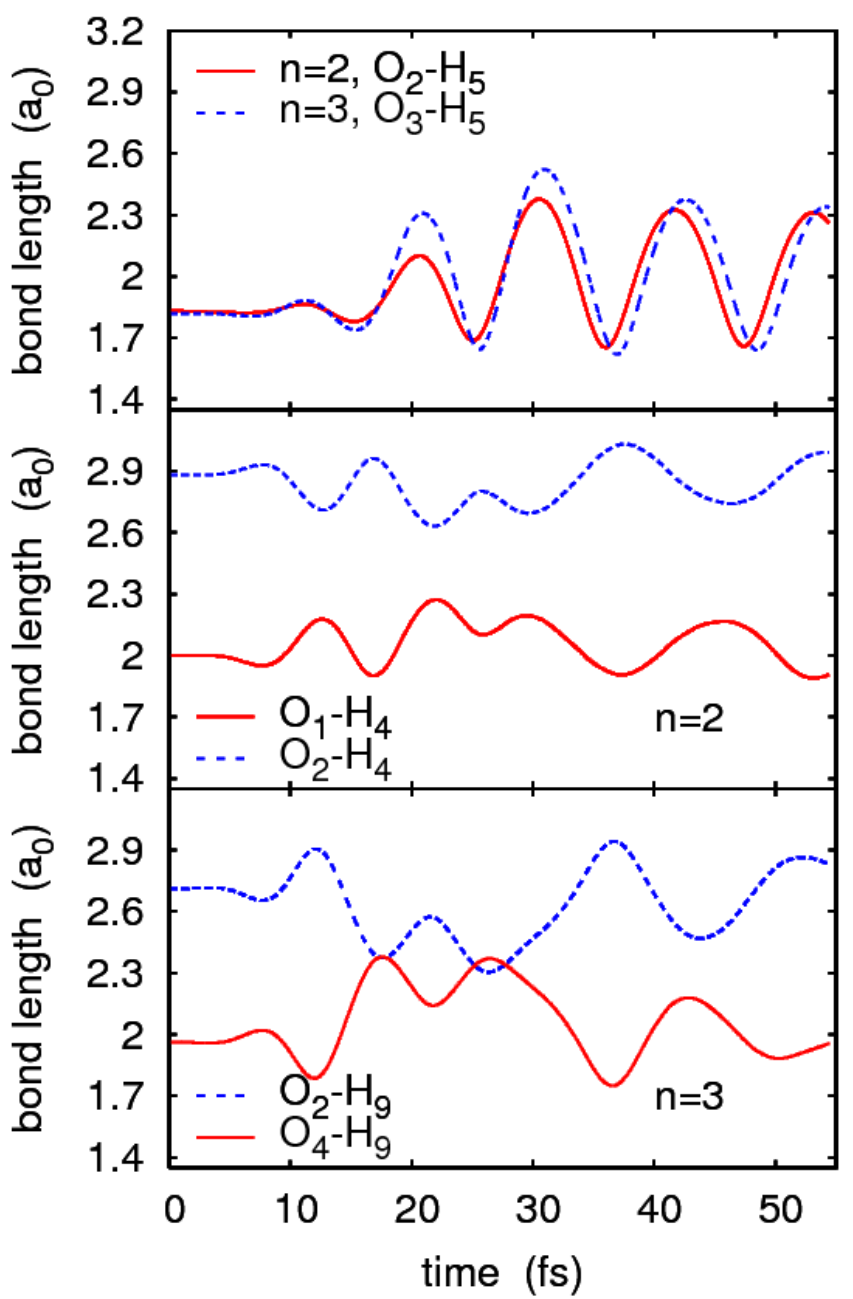

Fig. 6. Irradiation of $\left(\mathrm{H}_{2} \mathrm{O}\right)_{n} \mathrm{H}_{3} \mathrm{O}^{+}$, for $n=2,3$ by lasers with polarization along $x$ axis, FWHM of $20 \mathrm{fs}$, frequency of $0.48 \mathrm{eV}$, and intensity of $2.6 \times 10^{14} \mathrm{~W} / \mathrm{cm}^{2}$. Various $\mathrm{O}-\mathrm{H}$ bond lengths are depicted as a function of time, with labels according to the atom numbering of Figure 1

form the plot. The responses are remarkably similar. The middle panel focuses on $\left(\mathrm{H}_{2} \mathrm{O}\right)_{2} \mathrm{H}_{3} \mathrm{O}^{+}$internal $\mathrm{H}-\mathrm{O}-\mathrm{H}$ sequence comparing $\mathrm{H}-\mathrm{O}$ and $\mathrm{O}-\mathrm{H}$ vibrations, which turn out to be pretty similar and nicely in phase opposition, simply because the $\mathrm{O}$ atoms basically do not move. Finally the lower panel concerns $\left(\mathrm{H}_{2} \mathrm{O}\right)_{3} \mathrm{H}_{3} \mathrm{O}^{+}$, again focusing on a $\mathrm{H}-\mathrm{O}-\mathrm{H}$ sequence and with the same conclusions as in the middle panel.

\section{Conclusion}

We have explored in this paper the dynamical response of small water clusters (in particular $\left(\mathrm{H}_{2} \mathrm{O}\right) \mathrm{H}_{3} \mathrm{O}^{+}$) to a short and intense laser pulse for various frequencies and intensities covering the regime of stable vibrations up to Coulomb break-up of a highly ionized cluster. The first stages of response of the system are, as is well known from 
other systems, primarily taken up by the electrons and we have recovered this behavior. For low frequencies in the infra-red (IR), we also see a direct coupling of the laser pulse to the ionic centers of the clusters, particularly to the most mobile $\mathrm{H}^{+}$ions. This coupling becomes sizeable because the ionic vibrations are close to resonant conditions for IR frequencies. The effect is robust with respect to change of laser intensity, even for sizeable extra ionization up to typically one more charge unit. We have also studied larger hydrogen clusters with one or two more water molecules and found similar behaviors. We see here that the limits of stability against Coulomb break-up is shifted to higher ionization with increasing system size. The statements about stability have to be taken with care because our observation times are rather short. This means that we have discussed at least a stable transient stage. One needs to follow the evolution for longer time spans to find out more about the long-time stability. Work along that line is in progress.

This work was supported by the Deutsche Forschungsgemeinschaft (RE 322/10, RO 293/27), Fonds der Chemischen Industrie (Germany), a Bessel-Humboldt prize, a Gay-Lussac prize, the French Agence Nationale de la Recherche (ANR-06-BLAN0319-02), and the French computational facilities CalMip (Calcul en Midi-Pyrénées), IDRIS, CINES and CCRT.

\section{References}

1. Yulia V. Novakovskaya, Nikolai F. Stepanov, J. Phys. Chem. A 103 (1999) 3285.

2. M. D. Newton, J. Phys. Chem. 79 (1975) 2795.

3. H. Haberland, C. Ludewigt, H.-G. Schindler, D. R. Worsnop, J. Chem. Phys. 81 (1984) 3742.

4. R. N. Barnett, U. Landman, C. L. Cleveland, J. Jortner, J. Chem. Phys. 88 (1988) 4429.

5. O. Vendrell, F. Gatti, and H.-D. Meyer, Angew. Chem., Int. Ed. 46 (2007) 6918.

6. S. Tomoda, K. Kimura, Chem. Phys. Lett. 102 (1983) 560.

7. M. D. Newton, J. Chem. Phys. 67 (1977) 5535.

8. X. Yang, A. W. Jr. Castleman, J. Am. Chem. Soc. 111 (1989) 6845.

9. Z. Sternovsky, M. Horanyi, S. Robertson, Phys. Rev. A 64 (2001) 023203.

10. F. H. Stillinger, Science 209 (1980) 451.

11. O. Björneholm, F. Federmann, S. Kakar, and T. Möller J. Chem. Phys. 111 (1999) 546.

12. R.S. MacTaylor, A.W. Castleman Jr., J. Atmos. Chem. 36 (2000) 23.

13. S.S. Xantheas (Ed.), Recent Theoretical and Experimental Advances in Hydrogen-bonded Clusters, NATO Science Series C, vol. 561, Kluwer, Dordrecht, 2000.

14. M. Rapp, F.-J. Lubken, T.A. Blix, Adv. Space Res. 31 (2003) 2033.

15. K. D. Jordan, Science 306 (2004) 618.

16. B. Liu, S. Brøndsted Nielsen, P. Hvelplund, H. Zettergren, H. Cederquist, B. Manil, and B. A. Huber, Phys. Rev. Lett. 97 (2006) 133401.
17. L. Adoui, A. Cassimi, B. Gervais, J.-P. Grandin, L. Guillaume, R. Maisonny, S. Legendre, M. Tarisien, P. LópezTarifa, M.-F. Politis, M.-A. Hervé du Penhoat, R. Vuilleumier, M.-P. Gaigeot, I. Tavernelli, M. Alcamí, and F. Martín, J. Phys. B 42 (2009) 075101.

18. T. S. Zwier, Science 304 (2004) 1119.

19. M. P. Gaigeot, R. Vuilleumier, C. Stia, M. E. Galassi, R. Rivarola, B. Gervais, M. F. Politis, J. Phys. B 40 (2007) 1.

20. J. Kohanoff, E. Artacho, AIP Conf Proc 1080 (2008) 78.

21. U. Saalmann, C. Siedschlag, J. M. Rost, J. Phys. B 39 (2006) R39.

22. Th. Fennel, K.-H. Meiwes-Broer, J. Tiggesbäumker, P. M. Dinh, P.-G. Reinhard, E. Suraud, Rev. Mod. Phys. (2009) in press.

23. E. Runge, E. K. U. Gross, Phys. Rev. Lett. 52 (1984) 997.

24. E. K. U. Gross, W. Kohn, Adv. Quant. Chem. 21 (1990) 255.

25. M. A. L. Marques, E. K. U. Gross, Ann. Rev. Phys. Chem. 55 (2004) 427.

26. S. Goedecker, M. Teter, and J. Hutter, Phys. Rev. B 54 (1996) 1703.

27. Z. P. Wang, P. M. Dinh, P.-G. Reinhard, E. Suraud, in preparation.

28. J. P. Perdew and Y. Wang, Phys. Rev. B 45 (1992) 13244.

29. C. Legrand, E. Suraud, P.G. Reinhard, J. Phys. B 35, (2002) 1115.

30. F. Calvayrac, P.-G. Reinhard, E. Suraud, C.A. Ullrich, Phys. Rep. 337 (2000) 493.

31. P.-G. Reinhard, E. Suraud, Introduction to Cluster Dynamics (Wiley, New York, 2003).

32. M. D. Feit, J. A. Fleck, and A. Steiger, J. Comp. Phys. 47 (1982) 412.

33. G. Lauritsch and P.-G. Reinhard, Int. J. Mod. Phys. C 5 (1994) 65.

34. V. Blum, G. Lauritsch, J. A. Maruhn, and P.-G. Reinhard, J. Comp. Phys 100 (1992) 364.

35. R. Car and M. Parinello, Phys. Rev. Lett. 55 (1985) 2471.

36. M. Brack, Rev. Mod. Phys. 65 (1993) 677.

37. F. Calvayrac, E. Suraud, P.-G. Reinhard, Phys. Rev. B 52 (1995) R17056

38. K. Yabana and G. F. Bertsch, Phys. Rev. B 54 (1996) 4484.

39. F. Calvayrac, P.-G. Reinhard, E. Suraud, Ann. Phys. (NY) 255 (1997) 125.

40. Haibo Yu and Qiang Cui, J. Chem. Phys. 127 (2007) 234504

41. U. Näher, S. Björnholm, S. Frauendorf, F. Garcias, and C. Guet, Phys. Rep. 285 (1997) 245. 